\title{
Prognostic Factors in Terms of the Number of Metastatic Nodules in Patients With Colorectal Cancer Liver Metastases
}

\author{
Ki Ung Jang, Chan Wook Kim, Ki-Hun Kim, Seok-Byung Lim, Chang Sik Yu, Tae Won Kim, \\ Pyo Nyun Kim², Jong Hoon $\mathrm{Kim}^{3}$, Jin Cheon Kim \\ Department of Surgery, ${ }^{1}$ Department of Oncology, ${ }^{2}$ Department of Radiology and Research Institute of Radiology, and ${ }^{3}$ Department of \\ Radiation Oncology, Asan Medical Center, University of Ulsan College of Medicine, Seoul, Korea
}

Purpose: The hepatic resection is the gold-standard treatment for patients with colorectal-cancer liver metastases (CLM). This study aimed to identify prognostic factors in patients with synchronous CLM who underwent a surgical curative (R0) resection with respect to the number of metastatic nodules.

Methods: Of 1,261 CLM patients treated between January 1991 and December 2010, 339 who underwent a R0 resection for synchronous CLM were included in this retrospective analysis. Patients were grouped according to the number of CLM nodules: 1-2 CLM nodules, $\mathrm{n}=272$ (group 1) and 3-8 CLM nodules, $\mathrm{n}=67$ (group 2).

Results: The 5-year progression-free survival (PFS) rate in group 1was better than that in group $2(\mathrm{P}=0.020)$. The multivariate analysis identified lymph-node metastasis (N2), lymphovascular invasion (LVI), and three or more CLM nodules as independent poor prognostic factors for PFS in all patients and lymph-node metastasis (N2) and LVI as independent poor prognostic factors for patients in group 1 . No independent prognostic factors were identified for patients in group 2. CLM treatment method and neoadjuvant chemotherapy were not associated with survival.

Conclusion: Three or more metastatic nodules, lymph-node metastasis (N2), and LVI were independent poor prognostic factors for PFS in patients with synchronous CLM who underwent a R0 resection. The latter 2 factors were also independent prognostic factors for PFS in patients with less than 3 CLM nodules; however, in patients with three or more CLM nodules, the prognosis for PFS may be related only to liver metastasis.

\section{Keywords: Colorectal neoplasms; Liver metastasis; Metastatic nodules}

\section{INTRODUCTION}

The liver is the most common site of colorectal-cancer metastasis, which is found at the time of initial diagnosis in up to $25 \%$ of patients with colorectal cancer. A surgical curative resection (R0 resection) provides the best chance of long-term survival, with re-

Received: February 19, 2016 • Accepted: June 11, 2016

Correspondence to: Jin Cheon Kim, M.D.

Department of Surgery, Asan Medical Center, University of Ulsan College of Medicine, 88 Olympic-ro 43-gil, Songpa-gu, Seoul 05505, Korea

Tel: +82-2-3010-3489, Fax: +82-2-474-9027, E-mail: jckim@amc.seoul.kr

(C) 2016 The Korean Society of Coloproctology

This is an open-access article distributed under the terms of the Creative Commons Attribution NonCommercial License (http://creativecommons.org/licenses/by-nc/4.0) which permits unrestricted non-

commercial use, distribution, and reproduction in any medium, provided the original work is properly cited. ported 5-year survival rates of $35 \%-60 \%[1,2]$. However, only $10 \%-20 \%$ of patients with colorectal-cancer liver metastases (CLM) are candidates for a hepatic resection. Furthermore, even after a successful resection, $50 \%-70 \%$ of patients eventually suffer recurrence $[3,4]$. Despite this, the hepatic resection is the goldstandard treatment for patients with CLM, and when combined with perioperative chemotherapy, it is associated with an improvement in progression-free survival (PFS).

Advances in neoadjuvant therapy, which has rendered more patients resectable, together with improved perioperative outcomes, have extended the indications for surgical therapy for CLM [5-7]. Radiofrequency ablation (RFA) is also acceptable as a first-line therapy for some patients with CLM [8]. Compelling evidence exists that select patients with CLM have benefited from a R0 resection [9]; however, extending the indications for surgical ther- 
apy in patients with CLM has increased the clinical heterogeneity of these patients.

Although patients with CLM are uniformly grouped within the American Joint Committee on Cancer stage IV, they may have various disease characteristics that influence their prognosis. Therefore, several clinical scoring systems have been developed to predict individual outcomes more accurately; however, external validation of these scoring systems is limited, their clinical value remains controversial, and reliable prognostic factors have not yet been clearly identified $[10,11]$. Also, few studies have clearly demonstrated the prognostic value of the number of metastatic nodules. Therefore, the aims of the current study were to examine whether different clinical factors, namely, primary colorectal-cancer-related factors, metastatic-cancer-related factors, and modality of treatment, affected the PFS of synchronous CLM patients who underwent a R0 resection and to identify prognostic factors in such patients when grouped according to the number of metastatic nodules.

\section{METHODS}

\section{Patients and parameters}

CLM data collected in our institution over a 20 -year period (1991-2010) were reviewed retrospectively. Of 1,261 consecutive CLM patients treated by surgery, 339 (26.9\%) were included, and 922 (73.1\%) were excluded. Patients were eligible for this study if they had undergone curative-intent surgery for primary colorectal cancer and synchronous CLM. The study protocol was approved by the Institutional Review Board of Asan Medical Center (approval number: 2015-0063). This study was conducted in accordance with the guidelines in the Declaration of Helsinki.

Ablation of hepatic metastatic lesions included surgical resection or RFA, and patients who received neoadjuvant chemotherapy were included. The exclusion criteria were as follows: (1) a followup period < 2 years; (2) R1 or R2 resection (palliative resection or bypass surgery); (3) metachronous CLM; (4) age > 75 years; (5) > 8 CLM nodules; (6) PFS < 3 months; (7) a confirmed second primary cancer; (8) synchronous metastases at extrahepatic sites; and (9) patients who underwent RFA only because CLM was not confirmed histologically in those patients. Clinicopathological parameters included demographics, the number of CLM nodules, the diameter of the largest CLM nodule, the diameter sum of all CLM nodules, the follow-up duration, the preoperative serum carcinoembryonic antigen (s-CEA) level, histologic results, the location of the primary lesion, the operative method for the primary cancer, methods of CLM treatment (resection only or resection plus RFA), methods of liver resection (wedge resection [WR] or anatomical resection), and neoadjuvant chemotherapy.

The diameter of the CLM nodules was determined from pathology reports, preoperative imaging studies, or intraoperative ultrasonography. For each patient, the diameter of the largest CLM nodule and the sum diameter of all CLM nodules were divided by their mean values ( 2.6 and $3.3 \mathrm{~cm}$, respectively) for a simplified 2 -sided analysis. Pathologic results included the T stage, the $\mathrm{N}$ stage, the growth pattern of the primary tumor (expanding vs. infiltrative), the differentiation status of the primary tumor (lowgrade [well-differentiated or moderately-differentiated adenocarcinoma] vs. high-grade [poorly-differentiated adenocarcinoma, mucinous carcinoma, or signet-ring-cell carcinoma]), lymphovascular invasion (LVI) (positive vs. negative), and perineural invasion (positive vs. negative). The primary lesion was located in the colon or the rectum, and the method of CLM treatment was categorized as "resection only" or "resection plus RFA." For liver resection, a WR, an anatomical resection, or an anatomical resection combined with a WR were used. For preliminary patients' grouping, a receiver-operating-characteristic (ROC) analysis was used to divide patients into 2 groups according to the number of metastatic nodules associated with tumor recurrence based on the highest Youden index (sensitivity $=0.220$, specificity $=0.165$ ): group 1 (1-2 nodules, $\mathrm{n}=272)$ and group 2 (3-8 nodules, $\mathrm{n}=$ 67). Patients were also categorized according to preoperative sCEA levels, with the cut-off being the median value of $6 \mathrm{ng} / \mathrm{mL}$.

During a standardized postoperative follow-up (every 6 months for 5 years), documentation of recurrence was based on histologic confirmation or imaging studies, including dynamic computed tomography $(\mathrm{CT})$, magnetic resonance imaging, or positron emission tomography-CT. If recurrence was suspected or confirmed, the patients were followed up more frequently.

\section{Statistical analysis}

Categorical variables were compared using Pearson chi-square test or Fisher exact test. The Student $\mathrm{t}$-test was used to compare continuous variables between groups. The PFS and the overall survival (OS) rates were compared using the Kaplan-Meier method and log-rank testing. Potential variables were verified by using a multivariate analysis with the Cox proportional hazards model. Statistical significance was defined as a P-value $<0.05$. A ROC curve was used to define the threshold value for the number of CLM nodules associated with tumor recurrence. All calculations were performed using IBM SPSS ver. 18.0 (IBM Co., Armonk, NY, USA).

\section{RESULTS}

\section{Patient and tumor characteristics}

Table 1 shows the clinicopathologic features of all patients $(\mathrm{n}=$ $339)$ and of groups $1(n=272)$ and $2(n=67)$. The mean followup period for patients who underwent a R0 resection for synchronous CLM was 49.7 months. Fifteen patients (4.4\%) received a curative R0 resection after neoadjuvant chemotherapy. Group 2 patients had lower mean PFS and OS rates and shorter follow-up periods than group 1 patients $(\mathrm{P}=0.001, \mathrm{P}=0.001$, and $\mathrm{P}=0.002$, respectively). No statistically significant differences in the other clinicopathologic parameters were noted between groups 1 and 2, 
except for the use of neoadjuvant chemotherapy $(\mathrm{P}<0.001)$.

\section{Methods used to treat the primary colorectal cancer and CLM}

The surgical techniques used to treat the primary colorectal cancer and CLM are listed in Table 2. Primary colorectal cancer was treated according to the location of the tumor. No statistically significant differences in surgical treatment based on the location of the tumor were noted between groups 1 and 2. A review of the pa- thology reports confirmed the use of a $\mathrm{R} 0$ resection in all cases. Local treatments for CLM were resection only or combined resection and RFA. Although most patients (92.6\%) underwent a resection only, a significant difference in treatments was noted between groups 1 and 2; 264 patients (97.1\%) in group 1 underwent resection only and 17 patients (25.4\%) in group 2 underwent combined resection and RFA $(\mathrm{P}<0.001)$. For liver metastases, a WR was the most frequent method of CLM resection $(n=216,68.8 \%)$ whereas an anatomical resection was done in 82 patients $(26.1 \%)$.

Table 1. Patients' characteristics

\begin{tabular}{|c|c|c|c|c|}
\hline Characteristic & Total $(n=339)$ & Group 1 ( $n=272)$ & Group $2(n=67)$ & P-value \\
\hline Sex & & & & 0.19 \\
\hline Male & $209(61.7)$ & $163(59.9)$ & $46(68.7)$ & \\
\hline Female & $130(38.3)$ & $109(40.1)$ & $21(31.3)$ & \\
\hline Age (yr) & $57.0 \pm 10.0$ & $57.0 \pm 10.0$ & $58.0 \pm 8.0$ & 0.14 \\
\hline Number of CLM & $1.9 \pm 1.3$ & $1.3 \pm 0.5$ & $4.1 \pm 1.3$ & $<0.001^{*}$ \\
\hline Largest diameter of CLM (cm) & $2.7 \pm 2.1$ & $2.6 \pm 2.1$ & $3.0 \pm 2.1$ & 0.24 \\
\hline Sum diameter of CLM (cm) & $3.3 \pm 2.8$ & $2.9 \pm 2.4$ & $6.0 \pm 3.7$ & $<0.001^{*}$ \\
\hline Follow-up period (mo) & $49.7 \pm 35.5$ & $52.0 \pm 37.3$ & $40.2 \pm 25.0$ & $0.002^{*}$ \\
\hline Preoperative level of CEA (ng/mL) & $34.0 \pm 192.6$ & $37.0 \pm 213.7$ & $22.1 \pm 49.2$ & 0.57 \\
\hline T-stage & & & & 0.09 \\
\hline 0,1, or 2 & $20(5.9)$ & $13(4.8)$ & $7(10.4)$ & \\
\hline 3 or 4 & $319(94.1)$ & $259(95.2)$ & $60(89.6)$ & \\
\hline $\mathrm{N}$-stage & & & & 0.053 \\
\hline 0 & $88(26.0)$ & $64(23.5)$ & $24(35.8)$ & \\
\hline 1 & $146(43.1)$ & $117(43.0)$ & $29(43.3)$ & \\
\hline 2 & $105(30.9)$ & $91(33.5)$ & $14(20.9)$ & \\
\hline Bormann type & & & & 0.49 \\
\hline | or II (expanding type) & $248(73.2)$ & $201(73.9)$ & $47(70.1)$ & \\
\hline III or IV (infiltrative type) & $91(26.8)$ & $71(26.1)$ & $20(29.9)$ & \\
\hline Differentiation & & & & 0.24 \\
\hline WD/MD & $312(92.0)$ & $248(91.2)$ & $64(95.5)$ & \\
\hline PD/Muc/SRC & $27(8.0)$ & $24(8.8)$ & $3(4.5)$ & \\
\hline Presence of LVI & $128(37.8)$ & $102(37.5)$ & $26(38.8)$ & 0.63 \\
\hline Presence of PNI & 55 (16.2) & $40(14.7)$ & $15(5.5)$ & 0.38 \\
\hline Primary tumor location & & & & 0.78 \\
\hline Colon & $167(49.3)$ & $135(49.6)$ & $32(47.8)$ & \\
\hline Rectum & $172(50.7)$ & $137(50.4)$ & $35(52.2)$ & \\
\hline Neoadjuvant chemotherapy & $15(4.4)$ & $5(1.8)$ & $10(14.9)$ & $<0.001^{*}$ \\
\hline PFS (mo) & $34.7 \pm 38.0$ & $37.2 \pm 40.2$ & $24.5 \pm 25.3$ & $0.001^{*}$ \\
\hline OS (mo) & $51.9 \pm 35.5$ & $54.5 \pm 37.3$ & $41.5 \pm 24.6$ & $0.001^{*}$ \\
\hline
\end{tabular}

Values are presented as mean \pm standard deviation or number of patients (\%).

Group 1, number of liver metastases $\leq 2$; group 2, number of liver metastases $>2$ and $\leq 8$; CLM, colorectal-cancer liver metastases; CEA, carcinoembryonic antigen; WD, well differentiated; MD, moderately differentiated; PD, poorly differentiated; Muc, mucinous type; SRC, signet ring cell type; LVI, lymphovascular invasion; PNI, perineural invasion; PFS, progression-free survival; OS, overall survival.

${ }^{*} \mathrm{P}<0.05$. 
Volume 32, Number 3, 2016

\section{Analysis of survival and risk of recurrence according to clinicopathologic parameters}

In all, 178 patients (52.5\%) had died and 218 (64.3\%) had suffered

Table 2. Surgical methods for treating primary colorectal cancer and treatments for colorectal-cancer liver metastases (CLM)

\begin{tabular}{lccc}
\hline Variable & $\begin{array}{c}\text { Group 1 } \\
(\mathrm{n}=272)\end{array}$ & $\begin{array}{c}\text { Group 2 } \\
(\mathrm{n}=67)\end{array}$ & P-value \\
\hline Surgery for primary tumor & & & 0.08 \\
\hline RHC & $47(17.3)$ & $8(11.9)$ & \\
LHC & $7(2.6)$ & $2(3.0)$ & \\
AR & $66(24.3)$ & $23(34.3)$ & \\
\hline LAR & $90(33.1)$ & $16(23.9)$ & \\
ULAR & $42(12.4)$ & $14(20.9)$ & \\
APR & $21(7.7)$ & $3(4.5)$ & \\
\hline Hartmann/TPC/STC/TC & $13(4.8)$ & $1(1.5)$ & \\
Treatment for CLM & & & $<0.001^{*}$ \\
Resection only & $264(97.1)$ & $50(74.6)$ & \\
Wedge resection & $185(70.1)$ & $31(62.0)$ & \\
Anatomical resection & $71(26.9)$ & $11(22.0)$ & \\
\hline Wedge + anatomical resection & $8(3.0)$ & $8(16.0)$ & \\
Resection + RFA & $8(2.9)$ & $17(25.4)$ & \\
\hline
\end{tabular}

Values are presented as number of patients (\%).

Group 1, number of liver metastases $\leq 2$; group 2, number of liver metastases > 2 and $\leq 8$; RHC, right hemicolectomy; LHC, left hemicolectomy; AR, anterior resection; LAR, low anterior resection; ULAR, ultralow anterior resection; APR, abdominoperineal resection; Hartmann, Hartmann's procedure; TPC, total proctocolectomy; STC, subtotal colectomy; TC, total colectomy; CLM, colorectal-cancer liver metastases; RFA, radiofrequency ablation. ${ }^{*} \mathrm{P}<0.05$.

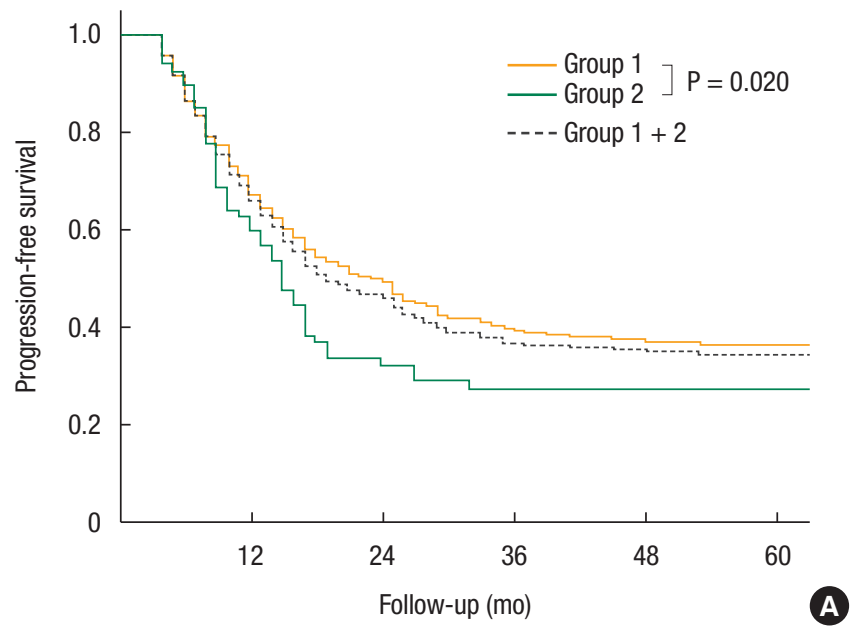

recurrence at the time of this retrospective analysis. The median OS period and PFS period were 59.0 and 19.0 months, respectively. The 1-, 3-, and 5-year OS rates for all patients who underwent a R0 resection for synchronous CLM were $97.6 \%, 68.2 \%$, and $48.3 \%$, respectively, and the 1-, 3-, and 5-year PFS rates were $65.9 \%, 37.1 \%$, and $34.6 \%$, respectively. The $1-, 3-$, and 5 -year OS rates were $97.0 \%, 69.7 \%$, and $49.4 \%$ in group 1 and $97.0 \%, 62.5 \%$, and $44.5 \%$ in group 2 , respectively. In addition, the 1-, 3-, and 5 -year PFS rates were $71.2 \%, 39.4 \%$, and $36.5 \%$ in group 1 and $59.7 \%, 27.7 \%$, and $27.7 \%$ in group 2, respectively. Fig. 1 shows the PFS and the OS curves for group 1, group 2, and all patients (group $1+$ group 2 ). A statistically significant difference in the PFS rate was noted between groups 1 and $2(P=0.047)$, but not in the $\mathrm{OS}$ rate $(\mathrm{P}=0.251)$.

Univariate analysis revealed that a preoperative s-CEA level $>6$ $\mathrm{ng} / \mathrm{mL}$, lymph node metastasis, and LVI were poor prognostic factors for PFS in all patients $(\mathrm{P}=0.007, \mathrm{P}<0.001$, and $\mathrm{P}=0.001$, respectively) and for patients in group $1(\mathrm{P}=0.009, \mathrm{P}<0.001$, and $\mathrm{P}=0.003$, respectively). The only significant parameter related to PFS in group 2 was LVI $(\mathrm{P}=0.036)$. The multivariate analysis identified the number of metastatic nodules, lymph node metastasis (N2), and LVI as independent prognostic factors for PFS in all patients $(\mathrm{P}=0.020, \mathrm{P}=0.007$, and $\mathrm{P}=0.004$, respectively). In addition, lymph node metastasis (N2) and LVI were identified as independent prognostic factors for PFS in group 1 (both $\mathrm{P}=$ 0.015). No independent prognostic factors for PFS were identified in group 2 (Table 3). The other clinicopathologic parameters (age, gender, $\mathrm{T}$ stage, Borrmann type, differentiation, perineural invasion, largest CLM nodule diameter, sum diameter of all CLM nodules, and method of CLM resection) that were evaluated in our analysis showed no correlation with the PFS of patients with CLM patients.

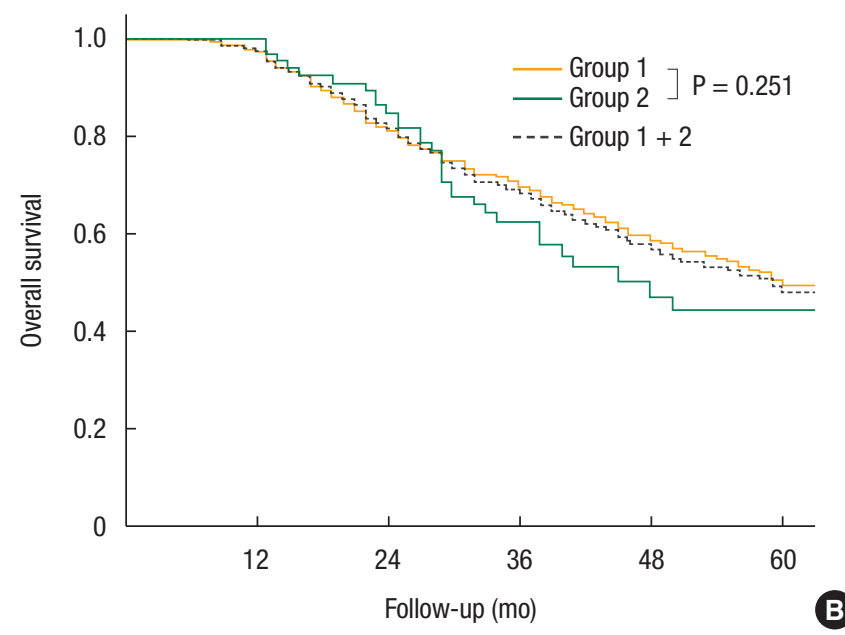

Fig. 1. Kaplan-Meier curves showing the progression-free survival (PFS) (A) and overall survival (OS) (B) in group 1, group 2, and both groups combined (group $1+2$ ). Group 1, number of liver metastases $\leq 2$; group 2, number of liver metastases $>2$ and $\leq 8$. 
Table 3. Univariate and multivariate analyses to identify clinicopathologic parameters associated with progression-free survival

\begin{tabular}{llllll}
\hline \multirow{2}{*}{ Variable } & \multicolumn{2}{c}{ Univariate } & & \multicolumn{2}{c}{ Multivariate } \\
\cline { 2 - 2 } \cline { 5 - 6 } & $\begin{array}{l}5 \text {-yr PFS } \\
\text { rate }(\%)\end{array}$ & P-value & & $95 \% \mathrm{Cl}$ & P-value \\
\hline
\end{tabular}

Group $1+2$

Number of CLM

$$
\text { Group } 1
$$

Group 2

Age (yr)

$$
\begin{aligned}
& \leq 60 \\
& >60
\end{aligned}
$$

Preoperative level of CEA $(\mathrm{ng} / \mathrm{mL})$

$$
\leq 6
$$

$>6$

$\mathrm{N}$-stage

N1

LVI

$\begin{array}{ll}\text { Negative } & 42 \\ \text { Positive } & 25.1\end{array}$

\begin{tabular}{|c|c|c|c|c|}
\hline$\leq 6$ & 43.3 & 1 & & \\
\hline$>6$ & 29.8 & 1.28 & $0.92-1.78$ & 0.14 \\
\hline $\mathrm{N}$-stage & & & & \\
\hline NO & 43.6 & 1 & & \\
\hline N1 & 45.9 & 1.01 & $0.65-1.58$ & 0.97 \\
\hline N2 & 19.7 & 1.75 & $1.11-2.75$ & $0.015^{*}$ \\
\hline LVI & & & & \\
\hline Negative & 44.9 & 1 & & \\
\hline Positive & 26.7 & 1.51 & $1.08-2.10$ & $0.015^{\star}$ \\
\hline
\end{tabular}

Group 1

Preoperative level of CEA (ng/mL)

PFS, progression-free survival; HR, hazard ratio; $\mathrm{Cl}$, confidence interval; $\mathrm{CLM}$, colorectal-cancer liver metastases; group 1, number of liver metastases $\leq 2$; group 2, number of liver metastases $>2$ and $\leq 8$; CEA, serum carcinoembryonic antigen; LVI, lymphovascular invasion.

${ }^{*} \mathrm{P}<0.05$.

\section{Analysis of survival according to the treatment method for CLM}

Survival rates for the treatment methods for CLM (resection only or resection plus RFA) were compared using the Kaplan-Meier method. No statistically significant differences between the two treatment modalities in terms of the PFS $(\mathrm{P}=0.688)$ or the OS (P
$=0.147$ ) rates were observed (Fig. 2).

Of the 339 patients who underwent a R0 resection for CLM, 30 patients $(8.8 \%)$ received preoperative chemotherapy. Fifteen of these patients $(4.4 \%)$ received preoperative chemo-radiation for low rectal cancer, and the other fifteen patients $(4.4 \%)$ received neoadjuvant chemotherapy for initially unresectable CLM. The Kaplan-Meier analyses for the OS and the PFS rates showed no statistically significant difference between the neoadjuvant chemotherapy group and the non-neoadjuvant chemotherapy group ( $\mathrm{P}=0.796$ and $\mathrm{P}=0.366$, respectively) (Fig. 2).

\section{DISCUSSION}

The management of and the prognosis for patients with CLM have been widely studied $[2,3,10-18]$. The aim of the current study was to identify prognostic factors for PFS following a R0 resection of synchronous CLM in terms of the number of metastatic nodules. Currently, no consensus exists regarding the indications for surgical resection of synchronous CLM. Therefore, this study was based on the assumption that a $\mathrm{R} 0$ resection of synchronous CLM would be the most important prognostic factor for survival in patients with CLM.

Various outcomes of a curative resection of synchronous CLM have been reported, with 5-year OS rates between 15\% and 50\% $[13,15,19-23]$. Compared to other studies, the 5-year OS rate in this cohort was relatively favorable at $48.3 \%$, which may be due to the improved quality of patient care as a result of the multidisciplinary treatment at our institution. Previous studies identified numerous prognostic indicators for CLM, including primary colorectal cancer stage $[6,18]$, tumor differentiation $[12,13,24]$, the size and the number of metastases [12, 15, 19, 23, 24], s-CEA levels $[6,14,16,18]$, the time to liver metastasis $[16,18]$, the presence of extrahepatic disease $[15,16]$, and other factors $[17,25]$. Our study identified the number of CLM nodules $(\geq 3)$, lymph node metastasis (N2), and LVI as poor prognostic factors for PFS in patients with synchronous CLM who underwent a R0 resection. These results reflect the fact that the prognosis for patients with CLM is affected by both the impact of hematogenous metastases and lymphatic metastases. According to our results on the prognostic implications of the number of CLM nodules, we recommend combined surgical strategies in patients with $<3$ CLM nodules.

Fujii et al. [26] reported that synchronous CLM in patients without lymph-node metastasis was a localized disease and that such patients had a good prognosis. Similar to the results of that study, our analysis suggests that the risk of recurrence in patients with N2-stage lymph-node metastasis is significantly higher than it is in patients with N0-stage lymph-node metastasis (hazard ratio [HR], 1.72; $\mathrm{P}=0.007$ ) (Table 3).

LVI has long been recognized as a poor prognostic factor for patients with colorectal cancer [27-30]. Our results have also shown that LVI has poor prognostic impacts on CLM patients. The lym- 

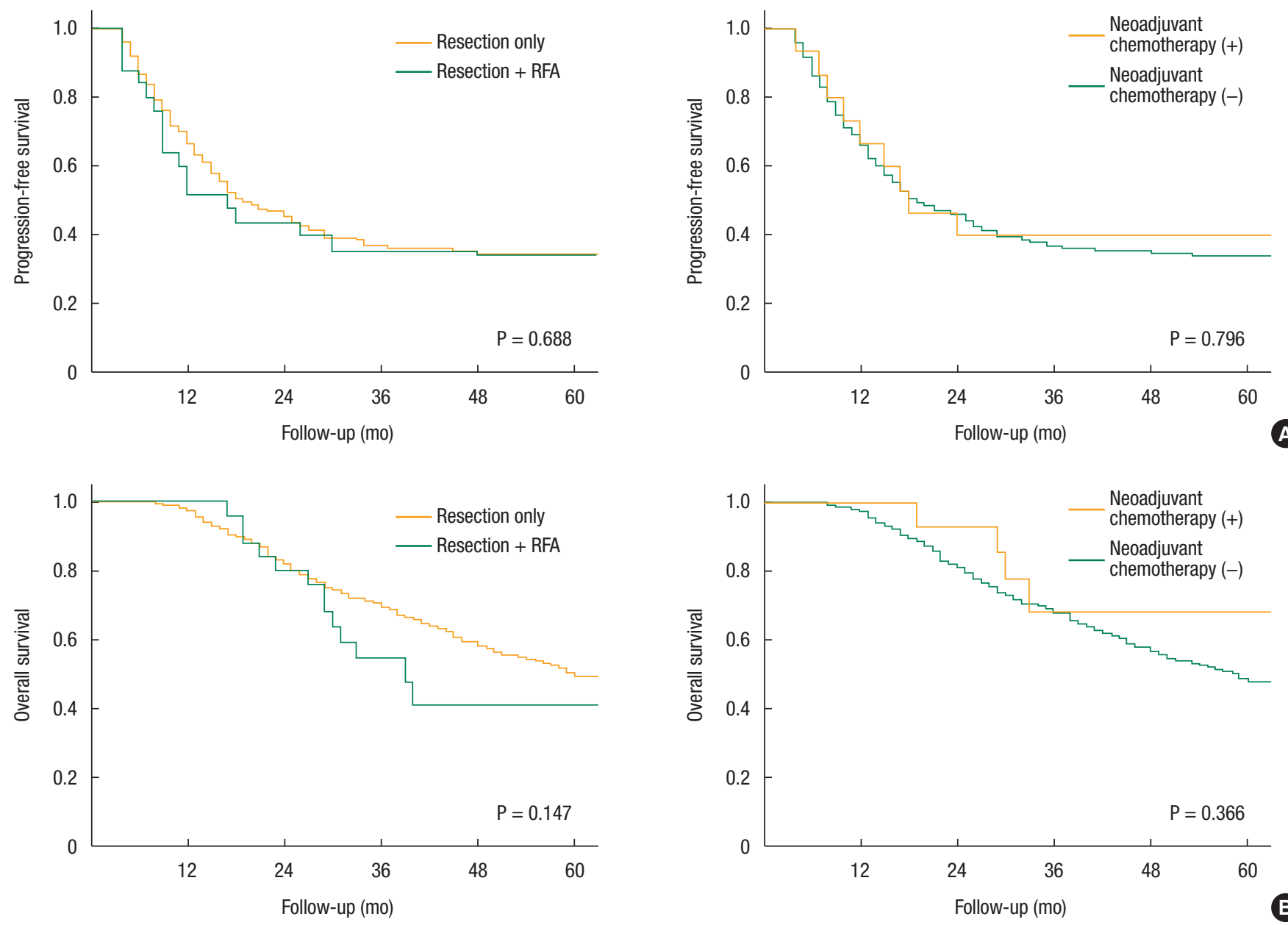

Fig. 2. Kaplan-Meier curves for the progression-free survival (PFS) (A) and overall survival (OS) (B) according to the treatment for colorectalcancer liver metastases. RFA, radiofrequency ablation.

phatic spread of tumor cells via lymphangiogenesis or pre-existing peritumoral lymphatics is an important early event in the metastasis to lymph nodes of a carcinoma, and this is true even in patients with CLM, although little is known regarding the exact mechanism [31].

The same prognostic factors (N2 stage and LVI) identified for the cohort as a whole were also identified specifically for patients in group 1, but no prognostic factors were identified specifically for group 2 patients (Table 3), indicating that a large number of metastatic nodules outweighs the effect of other prognostic factors. This effect may be explained by the dual blood supply of the liver (the hepatic artery and the portal vein). The large-scale trapping of colorectal carcinoma cells in the liver is dictated by the portal vein, which drains the mesenteric circulation directly into the liver [32]. Therefore, the effect of hematogenous metastases may be greater than that of lymphatic metastases in patients with a large number of CLM nodules.

In addition to the prognostic results for PFS, prognostic factors affecting OS were also analyzed. No statistically significant difference in OS was found between groups 1 and 2 patients (5-year OS rates: group 1, 49.4\%; group 2, 44.5\%) $(\mathrm{P}=0.251)$. Also, no prognostic factors for OS were found to differ between groups 1 and 2 . The independent poor prognostic factors for OS in all patients were (1) age $>60$ years (vs. $\leq 60$ years; $\mathrm{P}=0.049$; $\mathrm{HR}, 1.38$ ), (2) an $\mathrm{N}$ stage of N2 (vs. N0; P $<0.001 ; \mathrm{HR}, 2.33$ ), and (3) positive LVI (vs. negative LVI; $\mathrm{P}=0.001 ; \mathrm{HR}, 1.78$ ). Had there been a larger number of patients in group $2(n=67)$, the results of the prognostic analysis for OS might have been similar to those of the prognostic analysis for PFS.

In a previous study, cancer of the colon had a better prognosis than cancer of the rectum [33]; however, our risk analysis suggested that the location of the primary tumor had no significant effect on prognosis. This difference may be associated with the practice at our institution of strictly maintaining a total mesorectal excision and cylindrical resection during removal of rectal tumors. Retrospective reports and meta-analyses comparing the out- 
comes of patients who underwent a liver resection vs. a RFA have found better survival and less local intrahepatic recurrence after liver resection than after RFA [34-36]. In contrast, in another recent study, the 5-year OS rate in the group that received a resection only was $48 \%$, but the 5 -year OS rate in the group that received a RFA only was $69.8 \%$ [37]. We do not have any data comparing a resection only with a RFA because the RFA-only group was excluded from our analysis due to the high probability of over-staging in those patients because most patients in that group had not undergone confirmatory biopsies of hepatic lesions. However, patients that underwent a resection with RFA were included in the analysis and compared with patients that received a resection only, and no survival difference between the 2 groups was found (Fig. 2). Also, the method of CLM treatment was not a prognostic factor for PFS (Table 3). Twenty-five patients (7.4\%) underwent a resection with RFA, 17 of which (68\%) were in group 2. A RFA was indicated in patients with CLM with a maximum diameter of $3 \mathrm{~cm}$, in patients with CLM in locations that made resection technically difficult, or in patients for whom resection was contraindicated for other reasons. Based on the results of the comparison between resection only and resection plus RFA, the RFA may also be an alternative method for the ablation of CLM nodules in selected patients. Similar outcomes for intraoperative RFA as an adjunct to hepatic resection for the treatment of patients with CLM were reported in another recent study [38].

Patients who receive neoadjuvant chemotherapy to reduce the size of CLM show long-term OS comparable with that of patients with resectable primary tumors, despite widespread disease $[5,7$, $39,40]$. In the present study, 15 patients $(4.4 \%)$ who initially had unresectable CLM received neoadjuvant chemotherapy and a R0 resection. Of those who received neoadjuvant chemotherapy, 4 underwent a second operation (after the first operation to resect the primary colorectal cancer and neoadjuvant chemotherapy), and six received a targeting agent (cetuximab [Erbitux] or bevacizumab [Avastin]) combined with neoadjuvant chemotherapy. Patients received different neoadjuvant chemotherapy regimens during different cycles due to the long study period. Of the $15 \mathrm{pa}-$ tients that received neoadjuvant chemotherapy (neoadjuvant group), 11 (73.3\%) were alive and 6 (40.0\%) were progression-free at the end of the study. This group showed PFS and OS rates similar to those of the nonneoadjuvant group. However, because of the small number of patients in the neoadjuvant group and the different regimens used over the long study period, our study was unable to establish the efficacy of a R0 resection for the treatment of patients with synchronous CLM after neoadjuvant chemotherapy.

In conclusion, in this study, a high number of metastatic nodules $(\geq 3)$, lymph node metastasis (N2 stage), and LVI were independent poor prognostic factors for PFS in patients with synchronous CLM who underwent a R0 resection. Thus, we recommend surgical resection in patients with fewer than 3 CLM nodules. Lymph-node metastasis (N2) and LVI are also independent prognostic factors for patients with few hepatic metastases $(<3$ CLM nodules); however, in patients with multiple liver metastases ( $\geq 3$ CLM nodules), the prognosis may be related to liver metastasis alone. Therefore, we strongly recommend that patients with poor prognostic factors after undergoing a $\mathrm{R} 0$ resection for the treatment of CLM receive intensive chemotherapy and short-term surveillance and be closely monitored for disease recurrence.

\section{CONFLICT OF INTEREST}

No potential conflict of interest relevant to this article was reported.

\section{ACKNOWLEDGMENTS}

This study was supported by grants from our Institute for Life Sciences (2014-69), the Korea Research Foundation (2013070986) of the Ministry of Science, ICT, and Future Planning, the Korea Health 21 R\&D Project (HI06C0868 and HI13C1750), and the Center for Development and Commercialization of Anti-Cancer Therapeutics (HI10C2014) of the Ministry of Health and Welfare of the Republic of Korea.

\section{REFERENCES}

1. Kemeny N, Fata F. Arterial, portal, or systemic chemotherapy for patients with hepatic metastasis of colorectal carcinoma. J Hepatobiliary Pancreat Surg 1999;6:39-49.

2. Nathan H, de Jong MC, Pulitano C, Ribero D, Strub J, Mentha G, et al. Conditional survival after surgical resection of colorectal liver metastasis: an international multi-institutional analysis of 949 patients. J Am Coll Surg 2010;210:755-64, 764-6.

3. Adam R, Avisar E, Ariche A, Giachetti S, Azoulay D, Castaing D, et al. Five-year survival following hepatic resection after neoadjuvant therapy for nonresectable colorectal. Ann Surg Oncol 2001; 8:347-53.

4. House MG, Ito H, Gonen M, Fong Y, Allen PJ, DeMatteo RP, et al. Survival after hepatic resection for metastatic colorectal cancer: trends in outcomes for 1,600 patients during two decades at a single institution. J Am Coll Surg 2010;210:744-52, 752-5.

5. Leonard GD, Brenner B, Kemeny NE. Neoadjuvant chemotherapy before liver resection for patients with unresectable liver metastases from colorectal carcinoma. J Clin Oncol 2005;23:2038-48.

6. Nordlinger B, Guiguet M, Vaillant JC, Balladur P, Boudjema K, Bachellier P, et al. Surgical resection of colorectal carcinoma metastases to the liver. A prognostic scoring system to improve case selection, based on 1568 patients. Association Francaise de Chirurgie. Cancer 1996;77:1254-62.

7. Saltz LB, Clarke S, Diaz-Rubio E, Scheithauer W, Figer A, Wong R, et al. Bevacizumab in combination with oxaliplatin-based chemotherapy as first-line therapy in metastatic colorectal cancer: a randomized phase III study. J Clin Oncol 2008;26:2013-9.

8. Aliyev S, Agcaoglu O, Aksoy E, Taskin HE, Vogt D, Fung J, et al. 
Efficacy of laparoscopic radiofrequency ablation for the treatment of patients with small solitary colorectal liver metastasis. Surgery 2013;154:556-62.

9. Khatri VP, Petrelli NJ, Belghiti J. Extending the frontiers of surgical therapy for hepatic colorectal metastases: is there a limit? J Clin Oncol 2005;23:8490-9.

10. Reissfelder C, Rahbari NN, Koch M, Ulrich A, Pfeilschifter I, Waltert A, et al. Validation of prognostic scoring systems for patients undergoing resection of colorectal cancer liver metastases. Ann Surg Oncol 2009;16:3279-88.

11. Amano R, Yamada N, Nakata B, Kimura K, Yashiro M, Ohira M, et al. Prognostic indicator for the resection of liver metastasis of colorectal cancer. Surg Today 2014;44:1287-92.

12. Aoki T, Umekita N, Tanaka S, Noda K, Warabi M, Kitamura M. Prognostic value of concomitant resection of extrahepatic disease in patients with liver metastases of colorectal origin. Surgery 2008;143:706-14.

13. Arru M, Aldrighetti L, Castoldi R, Di Palo S, Orsenigo E, Stella M, et al. Analysis of prognostic factors influencing long-term survival after hepatic resection for metastatic colorectal cancer. World J Surg 2008;32:93-103.

14. Fong Y, Cohen AM, Fortner JG, Enker WE, Turnbull AD, Coit DG, et al. Liver resection for colorectal metastases. J Clin Oncol 1997;15:938-46.

15. Fong Y, Fortner J, Sun RL, Brennan MF, Blumgart LH. Clinical score for predicting recurrence after hepatic resection for metastatic colorectal cancer: analysis of 1001 consecutive cases. Ann Surg 1999;230:309-18.

16. Gayowski TJ, Iwatsuki S, Madariaga JR, Selby R, Todo S, Irish W, et al. Experience in hepatic resection for metastatic colorectal cancer: analysis of clinical and pathologic risk factors. Surgery 1994;116:703-10.

17. Nozoe T, Kohno M, Iguchi T, Mori E, Maeda T, Matsukuma A, et al. The prognostic nutritional index can be a prognostic indicator in colorectal carcinoma. Surg Today 2012;42:532-5.

18. Scheele J, Stang R, Altendorf-Hofmann A, Paul M. Resection of colorectal liver metastases. World J Surg 1995;19:59-71.

19. Beppu T, Sakamoto Y, Hasegawa K, Honda G, Tanaka K, Kotera Y, et al. A nomogram predicting disease-free survival in patients with colorectal liver metastases treated with hepatic resection: multicenter data collection as a Project Study for Hepatic Surgery of the Japanese Society of Hepato-Biliary-Pancreatic Surgery. J Hepatobiliary Pancreat Sci 2012;19:72-84.

20. Choti MA, Sitzmann JV, Tiburi MF, Sumetchotimetha W, Rang$\sin \mathrm{R}$, Schulick RD, et al. Trends in long-term survival following liver resection for hepatic colorectal metastases. Ann Surg 2002; 235:759-66.

21. Jaeck D, Bachellier P, Guiguet M, Boudjema K, Vaillant JC, Balladur $\mathrm{P}$, et al. Long-term survival following resection of colorectal hepatic metastases. Association Francaise de Chirurgie. Br J Surg 1997;84:977-80.

22. Jonas S, Thelen A, Benckert C, Spinelli A, Sammain S, Neumann
U, et al. Extended resections of liver metastases from colorectal cancer. World J Surg 2007;31:511-21.

23. Rees M, Plant G, Bygrave S. Late results justify resection for multiple hepatic metastases from colorectal cancer. Br J Surg 1997;84: 1136-40.

24. Gomez D, Morris-Stiff G, Wyatt J, Toogood G), Lodge JP, Prasad KR. Surgical technique and systemic inflammation influences long-term disease-free survival following hepatic resection for colorectal metastasis. J Surg Oncol 2008;98:371-6.

25. Yoshida D, Ikeda Y, Waki K, Shirabe K, Kakeji Y, Tsujitani S, et al. Different incidence of synchronous liver metastasis between proximal and distal colon cancer. Surg Today 2012;42:426-30.

26. Fujii T, Sutoh T, Morita H, Katoh T, Yajima R, Araki K, et al. Synchronous colorectal liver metastasis in patients without node metastasis: possibility of localized liver metastasis. Hepatogastroenterology 2013;60:1348-50.

27. Blumberg D, Paty PB, Picon AI, Guillem JG, Klimstra DS, Minsky BD, et al. Stage I rectal cancer: identification of high-risk patients. J Am Coll Surg 1998;186:574-9.

28. Koukourakis MI, Giatromanolaki A, Sivridis E, Gatter KC, Harris $\mathrm{AL}$; Tumour and Angiogenesis Research Group. Inclusion of vasculature-related variables in the Dukes staging system of colon cancer. Clin Cancer Res 2005;11(24 Pt 1):8653-60.

29. Lim SB, Yu CS, Jang SJ, Kim TW, Kim JH, Kim JC. Prognostic significance of lymphovascular invasion in sporadic colorectal cancer. Dis Colon Rectum 2010;53:377-84.

30. Minsky BD, Mies C, Rich TA, Recht A, Chaffey JT. Potentially curative surgery of colon cancer: the influence of blood vessel invasion. J Clin Oncol 1988;6:119-27.

31. Stacker SA, Achen MG, Jussila L, Baldwin ME, Alitalo K. Lymphangiogenesis and cancer metastasis. Nat Rev Cancer 2002;2: 573-83.

32. Valastyan S, Weinberg RA. Tumor metastasis: molecular insights and evolving paradigms. Cell 2011;147:275-92.

33. Kattan MW, Gonen M, Jarnagin WR, DeMatteo R, D’Angelica M, Weiser $\mathrm{M}$, et al. A nomogram for predicting disease-specific survival after hepatic resection for metastatic colorectal cancer. Ann Surg 2008;247:282-7.

34. Reuter NP, Woodall CE, Scoggins CR, McMasters KM, Martin RC. Radiofrequency ablation vs. resection for hepatic colorectal metastasis: therapeutically equivalent? J Gastrointest Surg 2009; 13:486-91.

35. Hur H, Ko YT, Min BS, Kim KS, Choi JS, Sohn SK, et al. Comparative study of resection and radiofrequency ablation in the treatment of solitary colorectal liver metastases. Am J Surg 2009; 197:728-36.

36. Wu YZ, Li B, Wang T, Wang SJ, Zhou YM. Radiofrequency ablation vs hepatic resection for solitary colorectal liver metastasis: a meta-analysis. World J Gastroenterol 2011;17:4143-8.

37. Kim KH, Yoon YS, Yu CS, Kim TW, Kim HJ, Kim PN, et al. Comparative analysis of radiofrequency ablation and surgical resection for colorectal liver metastases. J Korean Surg Soc 2011;81:25-34. 


\section{Coloproctology ki ung Jang, et al.}

38. Eltawil KM, Boame N, Mimeault R, Shabana W, Balaa FK, Jonker DJ, et al. Patterns of recurrence following selective intraoperative radiofrequency ablation as an adjunct to hepatic resection for colorectal liver metastases. J Surg Oncol 2014;110:734-8.

39. Brudvik KW, Bains SJ, Seeberg LT, Labori KJ, Waage A, Tasken K, et al. Aggressive treatment of patients with metastatic colorectal cancer increases survival: a scandinavian single-center experi- ence. HPB Surg 2013;2013:727095.

40. Nordlinger B, Sorbye H, Glimelius B, Poston GJ, Schlag PM, Rougier $\mathrm{P}$, et al. Perioperative chemotherapy with FOLFOX4 and surgery versus surgery alone for resectable liver metastases from colorectal cancer (EORTC Intergroup trial 40983): a randomised controlled trial. Lancet 2008;371:1007-16. 